\title{
Power Management and Handoff Evaluation of Mobile Telecommunication Infrastructure in Kuching, Sarawak
}

\author{
Taha Alhawas*, Umar Abdullah Saibukandu, Mujahid Tabassum \\ Swinburne University of Technology, Sarawak Campus, \\ Faculty of Engineering, Computing and Science \\ *Corresponding author email: tahambh@gmail.com
}

\begin{abstract}
Mobile networks are changing and incorporating new services continuously. The Telecommunication industry in East Malaysia is still under progressing mode. The terrain and tropical rainforest conditions are the biggest challenge to design a good telecommunication infrastructure in Sarawak. This might be the reason that the service level figures are even poorer in Sarawak and the demand on mobile networks in Sarawak is comparatively high. This paper contains a performance evaluation of mobile telecommunication infrastructure in Kuching. The research has focused on propagation issues and the findings are the result of several propagation tests that in various propagation conditions. Since the paper has been written for research purposes, the vendors' names in the study are not disclosed and are referred to as Provider A, B, and C. At the time of this research, only two vendors offered $4 \mathrm{G}$ services in Kuching.
\end{abstract}

Keywords: telecommunication, mobile networks, propagation and signals.

\section{Introduction}

Mobile networks have revolutionized the way people communicate and exchange data; they have fulfilled the everevolving desires of having the immediate ability to connect and interact with others wherever we are. Making mobile telephony and the internet readily available anywhere and anytime, require some of the most complex machines and systems ever produced. Together with a vastly intense research and development, mobile networks have been evolved from simple systems that offered basic texting services, data transmission and fully integrated multimedia communication to complex networks, sometimes considered as the stuff of science fiction.

The technological growth in the mobile industry is truly phenomenal and has attracted a vast number of subscribers around the world. In fact, aside from the internet, no other technology had ever witnessed such amount of growth achieved by mobile networks. Additionally, the technologies and services offered by mobile networks have changed drastically within a span of mere ten years. The need to cope with this rapid growth brought the issue of continuous development and efficient designs into focus. Furthermore, mobile networks need to maintain interoperability as they grow and incorporate new technologies, and network engineers need to stay at the leading edge of these technological advancements and the ever-increasing variety of offered mobile services.
The unprecedented growth of mobile networks requires sharp attention towards network planning, and persistent infrastructure development. Careful planning and constant development have become mandatory in a country like Malaysia, where the demand for mobile services is one of the highest in the world [1,2], as demonstrated in Figure 1 and Table 1.

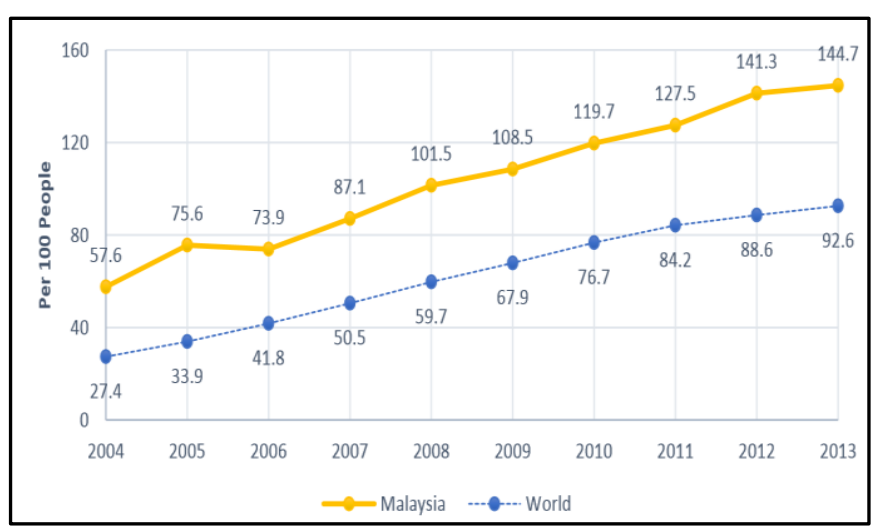

Figure 1. The Number of mobile subscriber per 100 inhabitants Malaysia vs. world [1,2]

Table 1. Demand comparison between Malaysia and other countries $[1,2]$

\begin{tabular}{|c|c|c|c|c|}
\hline Country & Malaysia & U.S & Japan & Worldwide \\
\hline $\mathbf{2 0 0 4}$ & 57.6 & 62.5 & 72.2 & 27.4 \\
\hline $\mathbf{2 0 0 5}$ & 75.6 & 68.3 & 76 & 33.9 \\
\hline $\mathbf{2 0 0 6}$ & 73.9 & 67.3 & 75.5 & 41.8 \\
\hline $\mathbf{2 0 0 7}$ & 87.1 & 82.1 & 84.4 & 50.5 \\
\hline $\mathbf{2 0 0 8}$ & 101.5 & 85.2 & 86.7 & 59.7 \\
\hline $\mathbf{2 0 0 9}$ & 108.5 & 88.6 & 91.3 & 67.9 \\
\hline $\mathbf{2 0 1 0}$ & 119.7 & 91.3 & 96.8 & 76.7 \\
\hline $\mathbf{2 0 1 1}$ & 127.5 & 94.4 & 102 & 84.2 \\
\hline $\mathbf{2 0 1 2}$ & 141.3 & 96 & 108 & 88.6 \\
\hline $\mathbf{2 0 1 3}$ & 144.7 & 95.5 & 115.2 & 92.6 \\
\hline
\end{tabular}

This research paper has been conducted in Sarawak, and mainly its capital city, Kuching. The terrain and climate of Sarawak present a bigger challenge to mobile network infrastructure planning. This might be the reason that the service level figures are even poorer in Sarawak. The demand to mobile networks in Sarawak is still comparatively high. In fact, Sarawak mobile subscriptions exceeded 108 per 100 inhabitants [2]. Therefore, a design that may have been considered modern and versatile a few years ago, will be outdated and inefficient if the system design does not take into account the growth potential of a certain market. 
Kuching as the capital city of Sarawak has almost $40 \%$ of the state's population; therefore, an improvement of the mobile network infrastructure is required. The terrain, environment, and climate of Kuching appear as an extra challenge for network planning. The signal propagation path can be heavily obstructed by thick foliage and dense trees. Normally, rain should not cause a propagation problem; however, rain conditions in Kuching are completely different from the rest of the state. In fact, Kuching is the wettest area in Malaysia. We suspect that such heavy rains may attenuate signals propagating at higher frequencies. The main objective of this research is to identify the current problem and the causes and to determine whether the current infrastructure needs improvement. Therefore, we have performed a survey and technical testing of mobile signal strength to determine the performance of the existing mobile network in various propagation conditions.

\section{Literature Review}

\subsection{Mobile Network Architecture}

A mobile network provides a wireless connection to the fixed Public Switched Telephone Network (PSTN) network, the basic elements needed to achieve this connectivity are [3]:

- Mobile Station MS: The user interface to the network, it is simply the handheld device used to access the network such as Mobile phone, Tablet, and ipads.

- Base Transceiver Station BTS: Often referred to as simply Base Station BS. This is the tower that provides coverage over a certain geographic area.

- Mobile Switching Centre MSC: This is the brain of the network, it coordinates all the BTSs connected to it and performs the call setup and management [3].

There are several other elements other than those discussed above, however the basic elements discussed here are common in all generations of mobile networks and they are responsible for the key tasks such as signal propagation and call setup. The rest of the elements usually are different in each generation and their tasks such as billing and account management are irrelevant to our project; therefore, they will not be discussed further.

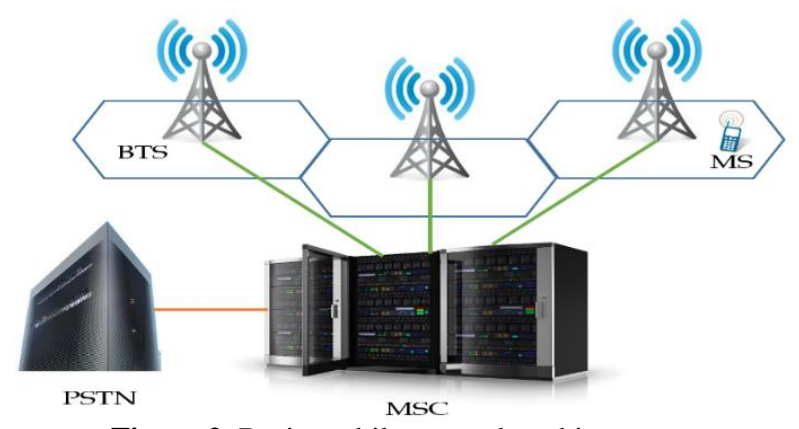

Figure 2. Basic mobile network architecture

\subsection{The Cellular Desing and Frequency Reuse}

Radio spectrum is a limited and tightly controlled the resource, therefore, it is necessary to consider the solutions that allow sharing of the radio spectrum among several users. The cellular design concept offers the solution by limiting the coverage of of each base station to a small geographic area, a cell. Each cell is assigned a set of frequencies that can be reused in co-channel cells in a different cell cluster as shown in Figure 3 [4].

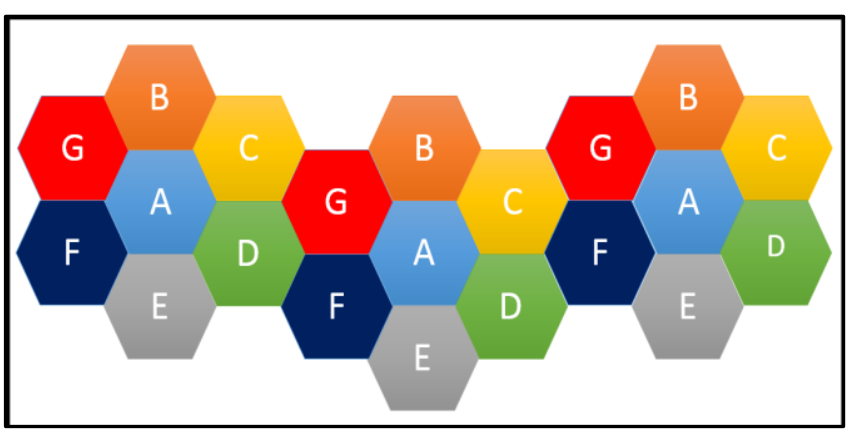

Figure 3. Cellular design

It is important to note that the hexagonal shaped cells shown above are only conceptual, cells in reality are neither hexagonal nor equal in size. This conceptual design allows for a limited cluster size, which needs to satisfy the equations [1]:

$$
N=i^{2}+i j+j^{2}
$$

The co-channel cells are determined for a 7-cell cluster as demonstrated in Figure $4[5,6]$.

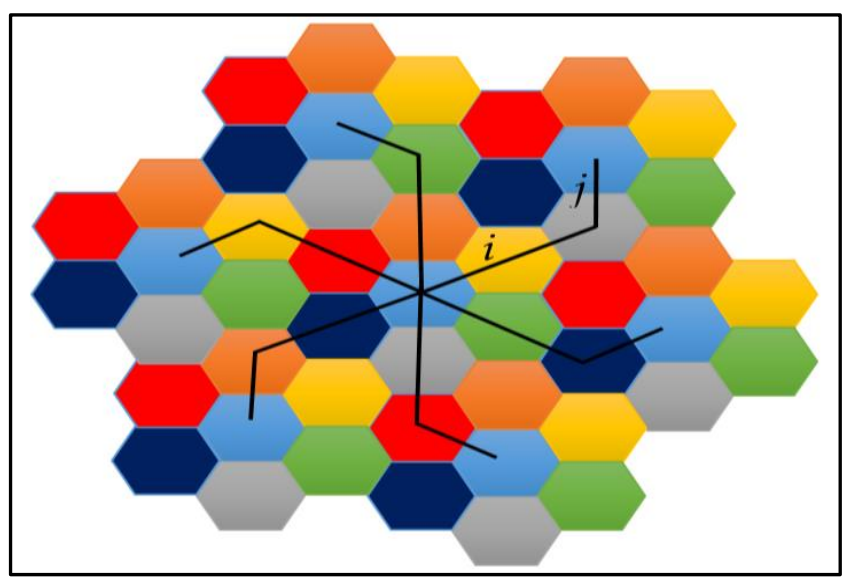

Figure 4. Locating co-channel cells in 7-cluster

\subsection{Propagation Mechanisms}

The simplest and most desired propagation mechanism is to have a direct line of sight path from the base station to the mobile station, unfortunately, in most cases the path will be obstructed by buildings, foliage, hills and obstruction. Therefore, it is important to adapt to other propagation mechanisms.

\subsubsection{Reflection}

Reflection is the change in direction of a wavefront at an interface between two different media, so that the wavefront returns into the medium from it was originated. In fact, even with a direct line of sight propagation, another version of the signal that is reflected from the surface of the earth will be received at the receiver with line of sight path, and the final received signal power is the sum of the two versions of the signal. It is important to note that waves are added vectorially and therefore, their sum may cause destructive or constructive interferences $[7,8]$. 


\subsubsection{Diffraction}

Diffraction is the phenomenon occurring when the radio wave is incident on objects with sharp edges such as building edges or tower tops. Similar to reflection, a diffracted version of the wave will be added vectorially with other versions received at the receiver $[7,8]$.

\subsubsection{Scattering}

If the propagation path has objects with dimensions comparable to the propagation wavelength, the radio wave will suffer from scattering. For example, millimeter waves have a prominent scattering effect in rain conditions.

Often, the final received signal power is the sum of many different versions of the signal, each with a different propagation path and mechanisms. Figure 5 illustrates a propagation scenario $[7,8]$.

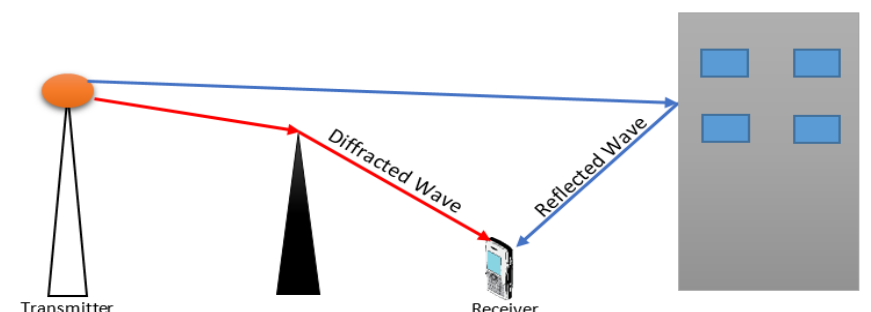

Figure 5. Multipath propagation scenario

\subsection{Call Handover Strategies}

Handover or handoff, is the process of automatically transferring a mobile station from one base station to another without hindering the quality or progress of the call. Handover must allocate the mobile station with new channels associated with the base station from which the call is to be handed over [9].

Several handover strategies exist, and they are all directly involved with base-station power control. The handover decisions were made by the MSC in early networks, whereby each base station continuously monitored the signal strength received by a MS and reported the obtained Received Signal Strength Indicator RSSI to the MSC. The MSC, being the brain of the network, decided whether a handover is necessary or not. In later networks, it was desired to relief the MSC from the burden and processing power needed for such decisions, therefore, an alternative approach was proposed whereby the MS itself monitors the signal obtained from all nearby base stations, and makes the handover decision if the received signal from a certain base station drops below a certain threshold. This strategy is better known as Mobile Assisted Handover MAHO [9].

Whether it is the MSC or the MS making the handover decision, the threshold value must be chosen carefully so that unnecessary handovers will not occur, and furthermore, the switching process must not cause call drops or quality degradation so that the whole process would be seamless and unperceivable by the user [9].

\subsection{Large-Scale Propagation}

When a mobile station moves away from the base station, the average received signal will gradually decrease. If the distance ranges from 5 to 40 propagation wavelengths, the phenomenon is called large-scale path-loss. There are statistical models that are capable of predicting the average signal strength at a certain distance from the base stations, those models are called large-scale propagation models and they only work in large MS-BS distances [8].

One very popular model is the Friis free space model which is used to predict the received power for signals propagating in free space. The signal power received at distance $d$ from the transmitter depends on a few factors such as [8]:

- $\quad$ The transmitter power $P_{T}$

- The Transmitter gain $G_{T}$

- The receiver gain $G_{R}$

- The propagation wave length $\lambda$

- The transmitter - receiver distance $d$.

And the received power at distance $\mathrm{d}$ is:

$$
P_{R}(d)=P_{T} G_{T} G_{r}\left[\frac{\lambda}{4 \pi d}\right]^{2}
$$

If the received power at a reference distance $d_{0}$ is measured as $P o$, the power at distance $d>d_{0}$ can be calculated using :

$$
\begin{array}{r}
P_{r}(d)=P_{0}\left[\frac{d_{0}}{d}\right]^{n} \text { Watts } \\
P_{r}(d) d B=P_{0}(d B)+10 n \log \left[\frac{d_{0}}{d}\right]^{n}
\end{array}
$$

where $n$ is the path loss exponent. Typical values of $n$ are stated in Table 2.

Table 2. Typical values of the path-loss exponent $n$

\begin{tabular}{|l|l|}
\hline Environment & N \\
\hline Free Space & 2 \\
\hline Urban Area & $2.7-3.5$ \\
\hline Shadowed Urban Area & $3-6$ \\
\hline Indoors Line of Sight & $1.6-1.8$ \\
\hline
\end{tabular}

\subsection{Log-Distance Propagation}

This model was developed to predict signal strength propagating indoors; it was found that the propagation indoors follows the power distance law.

It can be shown in Equation (5) as:

$$
P L(d B)=P L\left(d_{0}\right)+10 n \log \left(\frac{d}{d 0}\right)+X_{\sigma}
$$

where $X_{\sigma}$ is normal random variable in $\mathrm{dB}$ with $\sigma$.

\subsection{Small-Scale Fading}

The rapid fluctuation of a signal amplitude is known as Small-Scale fading or just fading, the effect of Small-Scale fading is much more severe than Large-Scale path loss. As mentioned earlier, there are several versions of the signal that may arrive at the receiver at slightly different times, those versions may add up destructively or constructively. Fading is caused by the destructive interference, and it is usually a temporary effect but occurs frequently. The rapid changes in signal strength over short distances is probably the most prominent effect of fading. Fading is not only caused by 
multiple path propagation, the speed at which the mobile station is travelling causes a Doppler shift in the propagation frequency, that will be different for each multipath component [8].

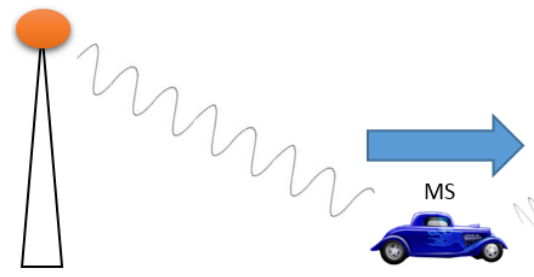

Figure 6. Doppler shift

As can be seen, the Doppler shift can be negative or positive depending on the direction of the MS movement. The frequency added to, or subtracted from the carrier frequency due to a Doppler shift is known as the actual Doppler shift and is defined as [3]:

$$
f_{d}=f_{c}-f_{R}
$$

where $f_{c}$ : is the carrier frequency and $f_{R}$ : is the received signal's frequency.

The Doppler shift depends on the velocity of the mobile stations as follows [3]:

$$
f_{d}=\frac{v}{\lambda} \cos (\theta)
$$

where $v$ : is the relative velocity of the mobile station, $\lambda$ : is the propagation wavelength, and $\theta$ : is the angle between the propagation path and the velocity vector.

\section{Experiments and Results}

In our research we tried to get as many user feedbacks as possible by conducting a questionnaire, and the responses were related to technical problems within the infrastructure of the network. After obtaining a sizable sample of users' feedback, we performed our signal strength testing to explain the questionnaire responses. Our concerns were with signal propagation issues, software and management issues.

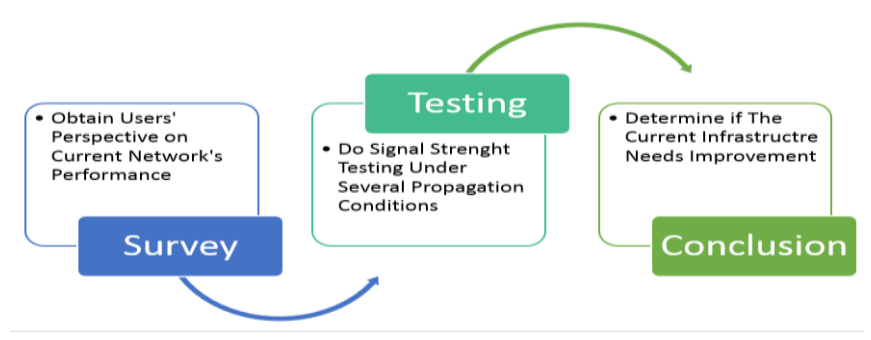

Figure 7. Experiment methodology

\section{Survey and Testing Results}

The majority of users reported that they use mobile internet services on their devices, most were getting a good 3G signal, and very few reported getting 4G. Many users reported that they experience service degradation during peak hours and heavy rain conditions and most of them reported that the signal quality was the same whether indoors or outdoors. Finally, about half of the users stated that they were experiencing call drops and slow internet speed while moving in a vehicle.

\subsection{Coverage}

Some users reported coverage issues; therefore, we have conducted some coverage testing for each vendor. According to our findings, the coverage is generally good in Kuching, there are however some dead zones where signal strength may drop significantly for each provider. For example, "provider A" had a dead zone illustrated by the unshaded areas in the heat map below.

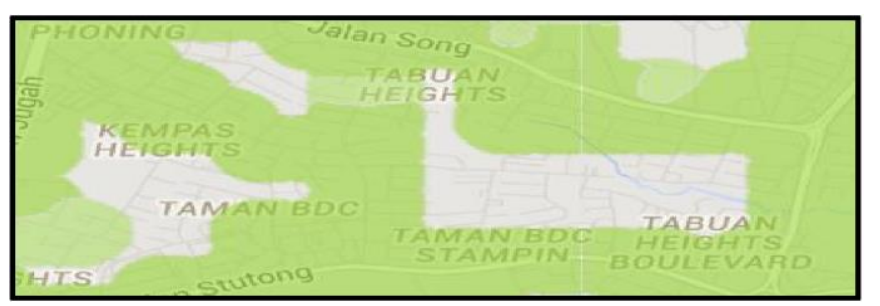

Figure 8. Some dead zones in the coverage map of provider A

In some severe cases, the signal drops to very low levels and becomes completely unusable. Similar results were found for all providers of interest in Kuching.

Providers have different coverage strategies, while Providers A and C relay on higher count of low power base stations, Provider B relies on lower count of high power base stations. Not all providers offer $4 \mathrm{G}$ coverage; however $4 \mathrm{G}$, is in fact available within the city.

\subsection{Service Consistency}

About $80 \%$ of our sample size reported service quality degradation during peak hours. We have done some signal strength testing for all major vendors during peak and offpeak hours, our findings are shown below in Figure 9.

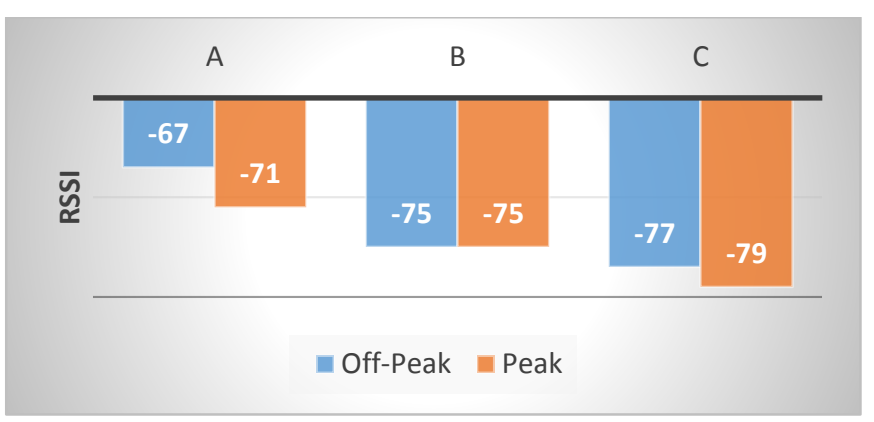

Figure 9. Peak vs Off-Peak Hours

\subsection{Heavy Rain Test}

About $63 \%$ of our sample reported service issues due to weather conditions, we carried measurements for $2 \mathrm{G}, 3 \mathrm{G}$, and $4 \mathrm{G}$ during heavy rain conditions, and the later was most severely impacted as shown below.

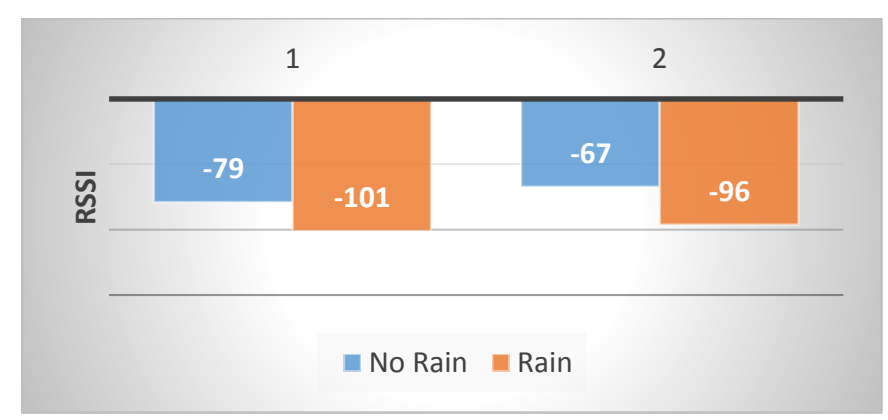

Figure 10. 4G Signal - Rain vs No rain 


\subsection{Building and Vehicle Penetration Test}

We carried a building and vehicle penetration tests since $40 \%$ of our sample reported receiving better signal outdoors. The results obtained are summarized below.

Indoors Outdoors In a Vehicle Outside a Vehicle

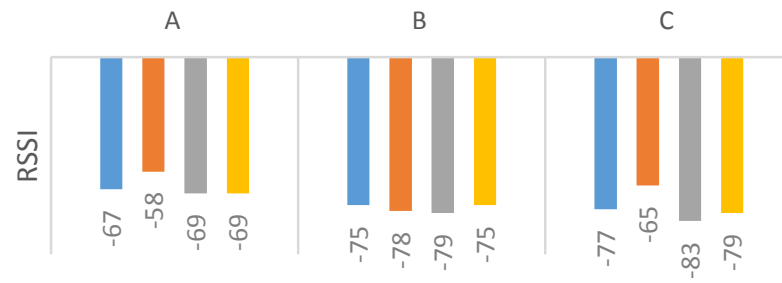

Figure 11. Building and vehicle penetration

The results show no real issue when it comes to building or vehicle penetration.

\subsection{Doppler Shift}

Since $41 \%$ of the sample size reported dropped calls or slow internet speeds while in a moving vehicle, we carried a Doppler shift test while moving in a vehicle towards an away from a base station at $70 \mathrm{~km} / \mathrm{h}$.

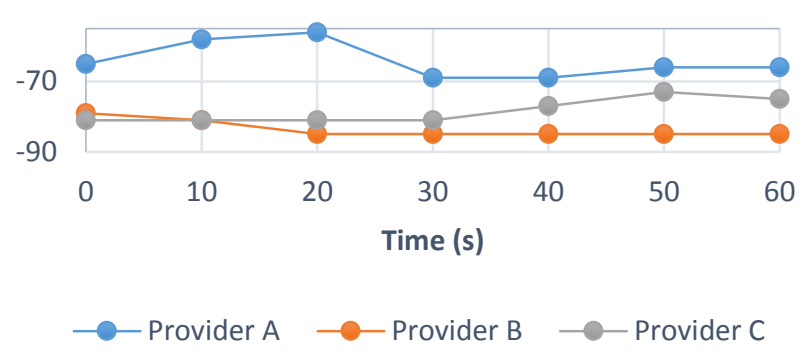

Figure 12. Doppler shift moving away from the BS

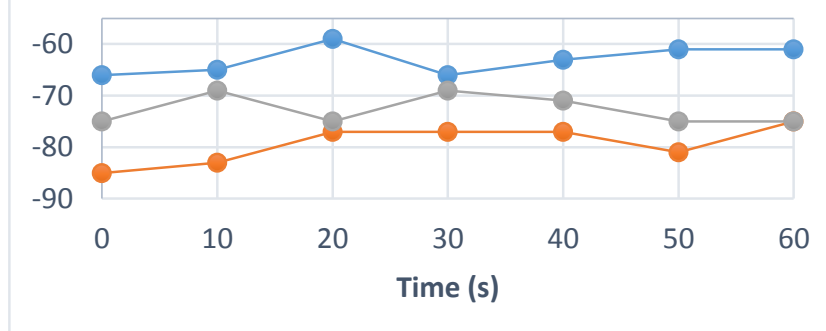

$\longrightarrow$ Provider A $\longrightarrow$ Provider B $\longrightarrow$ Provider C

Figure 13. Doppler shift moving toward the BS

According to our results shown in Figure 13, the signal strength for all carriers stayed within acceptable levels and there is no severe Doppler shift issue.

\subsection{Power Management and Handoff}

Dropped calls and signal loss can be a result of poor basestation power management or non-optimum handoff threshold. Call handoff must occur quickly and seamlessly. To test the handoff efficiency, we monitored the cell IDs as we drove around the city, and we registered the RSSI at which handoffs occurred. In general, handoff thresholds seem to be carefully chosen in Kuching, the signal never dropped below $-95 \mathrm{dBm}$ before a handoff occurrence, most mobile devices can operate without problems at $-95 \mathrm{dBm}$. Below is a sample of our test. Note that 10401, 10404, and 10406 represent the cell IDs.

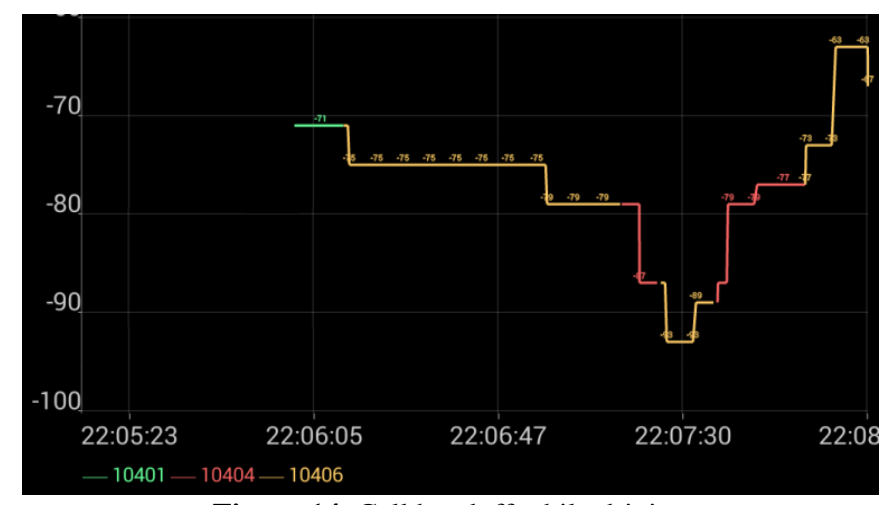

Figure 14. Call handoff while driving

Some areas outside the city, handoff and power management seem to be the issue, whereby handoffs occur even when the device is stationary, which causes call drops and very slow mobile internet speeds. The following figures show our testing results near that area. It can be seen that 10 handoffs occurred during 10 minutes while the device was stationary and there was no reason for a handoff to occur.

\begin{tabular}{|c|c|c|c|c|}
\hline Node & CELLID & Level & Qual & Type \\
\hline 41 & 10638 & -97 & - & $3 G$ \\
\hline 41 & 10627 & -99 & - & $3 G$ \\
\hline 41 & 10638 & -99 & - & $3 G$ \\
\hline & -- & -200 & -1 & -- \\
\hline & 1452 & -87 & -1 & $2 G$ \\
\hline & -- & -200 & -1 & -- \\
\hline & 19912 & -87 & -1 & $2 G$ \\
\hline 41 & 35643 & -93 & - & $3 G$ \\
\hline 41 & 10622 & -95 & - & $3 G$ \\
\hline 41 & 10633 & -95 & - & $3 G$ \\
\hline 41 & 10622 & -99 & - & $3 G$ \\
\hline & 1452 & -85 & -1 & $2 G$ \\
\hline & -- & -200 & -1 & -- \\
\hline & 18572 & -89 & -1 & $2 G$ \\
\hline 41 & 16382 & -99 & - & $3 G$ \\
\hline
\end{tabular}

Figure 15. Testing near Kuching central

\subsection{Additional Testing}

We carried some signal strength testing at the areas far from the city center and compared them with measurements taken in the city center. The results are shown in Figure 16 below.

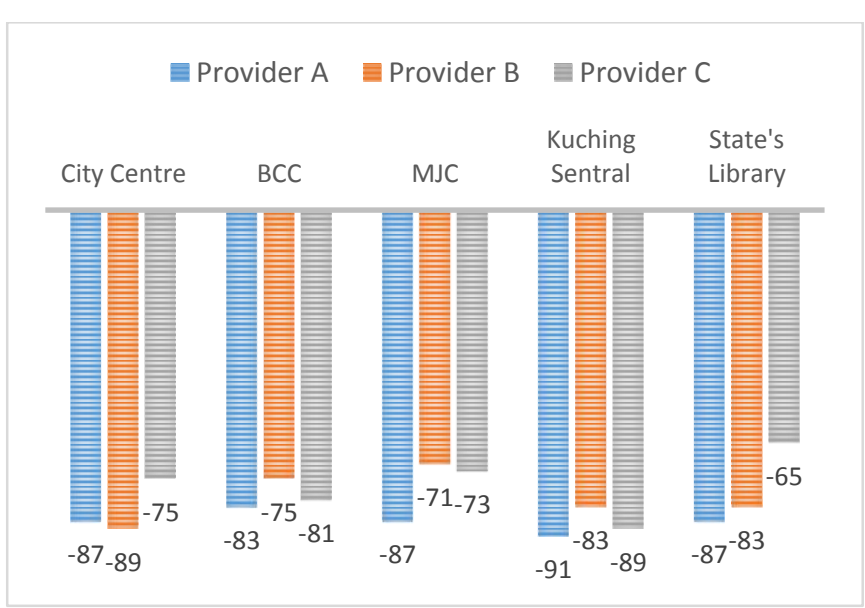

Figure 16. Additional signal strength tests

As evident from the results above, even far away from the city center, the signal strength stays within acceptable levels. 


\section{Discussion and Recommendations}

In our tests, we did observe weak signal strength outside and inside of the city, however the RSSI obtained from all providers stayed within acceptable levels unless we were testing inside a dead zone. It seems to be consistent in that area with the survey results whereby $92 \%$ of the responses reported average or better signal strength.

The approach to eliminate the coverage issues experienced by some users will depend on their usage details and the size of the area whereby coverage improvement is required. If the users are mainly using mobile internet, a directional antenna can enhance the signal strength. If the dead zone area is very small, then the user of carefully places reflectors can extend the coverage of a base station to include the dead zone. If it is large, there is no other option than adding a small base station to cover its area. Increasing the power of the base station nearest to a small dead zone may solve the coverage problem, but this may raise interference issues if not done carefully.

$4 \mathrm{G}$ is available within the city, users who are not getting $4 \mathrm{G}$ signal may not use $4 \mathrm{G}$ capable devices or have not activated $4 \mathrm{G}$ services.

The off-peak and peak-hours tests were relatively consistent, this point tells us that there are no signal propagation issues during peak hours. Any performance problems experienced by subscribers are likely related to server congestion and administration issues. Increasing the bandwidth will help significantly during congestion times, however; this may not always be possible and providers may need to use smaller cells so that only the users within the coverage area of the cell will share the bandwidth. Cell sectoring techniques could also be helpful.

As we suspected, the $2 \mathrm{G}$ network was unaffected by rain conditions, 3G was also stable, in fact; even $4 \mathrm{G}$ was mostly stable, only on very heavy rain conditions we started to observe a significant fall in signal levels. The wavelength of a $4 \mathrm{G}$ signal is around $11 \mathrm{~cm}$ and will not be attenuated by normal or even moderate rain conditions. Very heavy rains may cause destructive scattering. $4 \mathrm{G}$ is not exclusive to 2.6 $\mathrm{GHz}$ bands and can operate at 1800, 1900, 2100, and 2300 $\mathrm{MHz}$ bands [10]. Using lower frequency bands will limit the bandwidth slightly; however, it will guarantee a much more stable propagation. Some providers outside of Malaysia offer $4 \mathrm{G}$ at all bands mentioned above and the users can choose which band to connect to. Such solution is highly desired and recommended.

We noticed that $4 \mathrm{G}$ only works for data services, no calls can be made using $4 \mathrm{G}$ by either providers, upon further investigations, we found out that those providers are using $4 \mathrm{G}$ as data only network, and to support voice calls, they use a technique called Circuit Switched Fall-Back CSFB which switches the device momentarily to $3 \mathrm{G}$ or $2 \mathrm{G}$ during calls [10].

Our tests showed that for all services providers and using any network generation, the mobile signal has no real issue penetrating buildings and vehicles. Propagation outdoors is still better than indoors; in both cases, however the signal levels stayed at usable levels. This result can be attributed to the effective duplexing techniques, which has a major effect on penetration performance.

In our tests, the RSSI maintained a good level; the lowest propagation frequency of a mobile signal is $900 \mathrm{MHz}$, which makes Doppler shifts at $70 \mathrm{~km} / \mathrm{h}$ speed non-significant. The results matched our prediction; we further suspect that Doppler shifts will be significant at speeds upwards of 200 $\mathrm{km} / \mathrm{h}$. There are no high-speed trains in Sarawak, and the speed limit is $70 \mathrm{~km} / \mathrm{h}$ for cars; therefore, we do not think that providers should invest in combating Doppler shifts at high speeds. Users who reported service degradation while being mobile may have been handed over to a more congested base station or a lower capacity cell. Not much can be done in those cases other than increasing the capacity of congested areas. This can be achieved by using a layout with more base stations in congested areas.

We monitored the received RSSI while driving around the city for over 30 minutes; our devices were always being handed over before reaching RSSI levels lower than -95 $\mathrm{dBm}$. This suggests an optimal power management and handoff threshold implementation within the city in general. However, some areas such as Kuching central and pending in particular seems to have a problem whereby devices will be handed over while stationary. From the signal levels obtained in Figure 14, we can see that the signal was actually weak there. We exempted a power management problem is causing this behavior, and it seems that mobile stations cannot find good signal levels from any nearby base station, so they keep being handed over in an attempt to get a better signal. In any case, further investigation are needed to assess the problem effectively.

\section{Conclusion}

In the end, we conclude that the coverage of cellular network architecture in Kuching city was quite good, but some areas of the city had handoff and power management issues, whereby handoffs occur even when the device is stationary. There are some dead zones which are unavoidable due to the dense foliage. Therefore, the coverage plan must account for interference. The network did not suffer from major propagation issues during peak hours, so the problems of service degradation can be attributed to limited bandwidth or server congestions. The network performed optimally during rainy conditions and $4 \mathrm{G}$ was only impacted in very heavy rains. The signal did not have issues penetrating building or vehicles, moreover, there was no Doppler shift related issues as well. Handover occurred efficiently in most of our tests, but the power management problem was found in certain areas which caused signal and performance degradation. This may be considered by service providers to offer a better service to end users.

\section{References}

[1] N.a, "Mobile cellular subscriptions Data", International Telecommunication Union, [ONLINE] Available at: http://data.worldbank.org/indicator/IT.CEL.SETS. 
[2] N.a, "Suruhanjaya Komunikasi dan Multimedia Malaysia (SKMM) - Statistics, 2014", "Malaysian Communications And Multimedia Commission (MCMC)", [ONLINE] Available at: http://www.skmm.gov.my/Resources/Statistics.aspx

[3] Theodore S. Rappaport, "Wireless Communications: Principles and Practice (Prentice Hall Communications)", E. Edition. Prentice Hall, 2001.

[4] Ajay R. Mishra, "Fundamentals of Cellular Network Planning and Optimisation: 2G/2.5G/3G... Evolution to 4G”, 1 Edition, Wiley, 2004.

[5] Andre Perez, "Mobile Networks Architecture" 1 Edition, Wiley-ISTE, 2012.
[6] Paul Bedell, "Cellular Networks: Design and Operation A Real World Perspective", Edition, Outskirts Press, 2014.

[7] Abdollah Ghasemi, "Propagation Engineering in Wireless Communications", Springer, 2011.

[8] N. a, "2MULTIPLE-ACCESS TECHNIQUES", [ONLINE] Available at: http://www.wtec.org/loyola/wireless/02_04.htm.

[9] Martin Sauter, 2014. From GSM to LTE-Advanced: “An Introduction to Mobile Networks and Mobile Broadband", Revised Second Edition, Wiley.

[10]N. a, "LTE" [ONLINE] Available at: http://www.3gpp.org/technologies/keywordsacronyms/98-lte. 